\title{
Model-based synchronous machine control with compensation of model inaccuracies and instantaneous flux weakening capabilities
}

\author{
G. Zanuso*, L. Peretti*, P. Sandulescu* \\ ${ }^{\star} A B B$ Corporate Research, department of Electrical Systems, Forskargränd 7, 72178 Västerås, Sweden \\ [giovanni.zanuso], [luca.peretti], [paul.sandulescu]@se.abb.com
}

Keywords: synchronous machines, current regulation, flux weakening, non-linear models, parameter estimation.

\begin{abstract}
This paper proposes a model-based current/torque control strategy for synchronous machines, where the magnetic model mismatches are compensated in real time and the flux weakening operation is obtained without the need of an external voltage regulation loop. Starting from an initial (and potentially wrong) knowledge of the non-linear current-to-flux linkage relation in the form of a look-up-table, a combination of feedforward actions, compensating mechanisms and binary searches are used to achieve the target during real-time operation. The algorithm is implemented in a laboratory test bench and successfully verified with experiments on an 11-kW synchronous reluctance machine, proving the feasibility of the proposed approach.
\end{abstract}

\section{Introduction}

In the last decades, the development of synchronous machine drives came along with a wave of control approaches with increasingly complex algorithms, aimed to exploit the machine at its best static and dynamic performances possibly for a wide range of applications. Starting from the simple algorithms for surface permanent magnet synchronous machines (SPMSM), successful attempts were made to exploit the reluctance torque in interior permanent magnet synchronous machines (IPMSM), permanent magnet-assisted synchronous reluctance machines (PMaSynRMs) and synchronous reluctance machines (SynRMs) by using magnetic circuit model information. Today it is widely accepted that such information is required to achieve better performances. In particular, the knowledge on how the currents relate to the flux linkages, considering the effects of magnetic saturation and crossmagnetisation, is of foremost importance for the tuning of the regulators and the selection of the correct current references when a specific torque is required $[1,2]$.

Parallel efforts have been made towards the development of flux-weakening control schemes, in order to fully exploit synchronous machines wide speed ratio. Regulation-based approaches using the produced voltages as feedback have been proposed, as well as less conventional attempts to seamlessly integrate maximum-torque-per-ampere algorithms and fluxweakening algorithms [3, 4, 5]. Although many steps have been made towards robust solutions, there is still space for improvement in the area.

In addition to the classic problems of machine control, recent efforts have been directed to the development of control structures which are able to counter-act some model parameter mismatches $[6,7,8]$. Such mismatches could be the result of an improper identification of the machine magnetic model, or the deviation from the nominal flux linkage of the magnets due to temperature variations. Similar to the flux-weakening case, a final answer to this problem has not been reached yet.

On a global perspective, the efforts aimed at the development of robust algorithms for converter-driven synchronous machines should consider that the mass-production of machines of the same type and power range do not ensure that the machines have the same magnetic model, due to the inevitable differences introduced in the manufacturing process [9]. Therefore, the strive towards superior performances of synchronous machine drives should embrace both the improvement of the classic machine control problems (maximum efficiency, flux weakening) and the handling of parameter mismatches during real-time operation. Of course, this requires the drive to be equipped with initial information on the machine magnetic characteristics, obtained either in a manual or in an automatic fashion $[10,11,12]$. Summarising, it is expected that a synchronous machine drive should 1) perform at least maximum-torque-per-ampere (MTPA) operation within the constant torque region of the machine; 2) change smoothly and seamlessly towards flux weakening operation when required; 3) behave robustly and possibly compensate parameter errors, in presence of model mismatches.

This paper aims to demonstrate that these three goals could be achieved by the keen exploitation of an initial (and potentially wrong) knowledge of the non-linear magnetic model. MTPA and flux weakening are seamlessly integrated with no need of an external voltage loop control. At the same time, real-time changes of the magnetic model are monitored and compensated, ensuring a correct flux weakening behaviour in any condition. The target is achieved by calculating the current and voltage references by means of nested binary searches executed within one control cycle.

The paper is divided as follows. Section 2 describes the basics of the proposed control approach, focusing on the interaction of the different blocks. Section 3 reports the experimental results obtained on an 11-kW SynRM prototype, showing the most significant limitation cases during load operation. Section 4 includes some final remarks, followed by the references. 


\section{The control structure at a glance}

The principle of operation of a conventional field-oriented control (FOC) for a synchronous machine, with a cascaded structure of speed regulation and torque (or current) regulation, is the starting point of discussion for this paper. While the speed regulation part is irrelevant to this work, it is assumed that a torque reference signal, named $\tau^{*}$, is available. Such torque reference could be obtained, for example, as the output of a conventional speed PI regulation. The current/torque regulation part is now modified as shown in Figure 1, which reports an overall view of the proposed structure. The space vector notation (bold font) is introduced for currents, voltages and flux linkages.

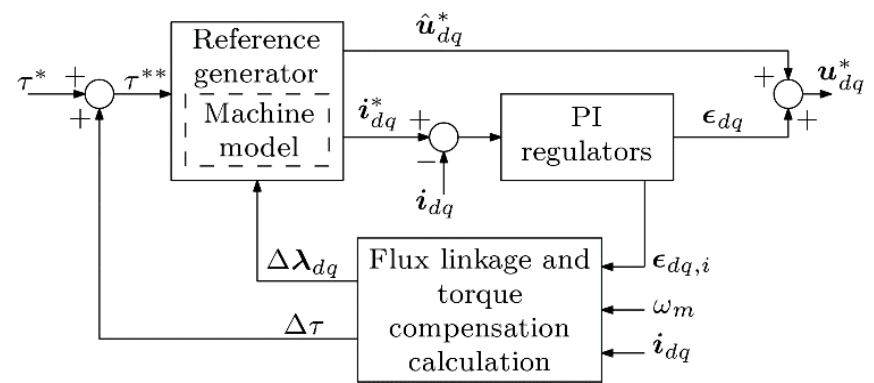

Figure 1: Schematic of the proposed control algorithm.

The torque reference $\tau^{*}$ is possibly modified by a torque compensation contribution $\Delta \tau$ which will be explained later in Subsection 2.3. The resulting modified torque reference $\tau^{* *}$ is sent to a reference generator, described in Subsection 2.4. The reference generator contains a machine model, which will be briefly discussed in Section 2.1. The output of the reference generator is composed by two current references for the rotating $d q$ reference frame of a synchronous machine $\left(\boldsymbol{i}_{d q}^{*}\right)$ and two estimated reference voltages for the same $d q$ frame $\left(\widehat{\boldsymbol{u}}_{d q}^{*}\right)$. The current references are compared to the measured currents $\boldsymbol{i}_{d q}$, and the resulting error is processed by conventional PI regulation blocks, performing a zero-steadystate-error control of the $d q$ currents. The output of the PI regulators $\boldsymbol{\epsilon}_{d q}$ is used as a compensation for the estimated reference voltage $\widehat{\boldsymbol{u}}_{d q}^{*}$. This action, known as feedforward especially in servo-motor applications, improves the current tracking by providing a pre-calculated trajectory for the voltages. At the same time, it unloads the PI regulation of the burden of producing the whole voltage vectors. What is typically neglected, but very relevant for the outcome of this work, is the information on the magnetic model mismatches contained in the PI regulators output, which could be exploited for a real-time adjustment of the flux linkage and torque estimation. The information, indicated as $\boldsymbol{\epsilon}_{d q, i}$ (see Subsection 2.2 ), is further processed by the flux linkage and torque compensation block of Figure 1. This block, detailed in Subsection 2.3, generates the torque compensation mentioned above and a flux compensation $\Delta \lambda_{d q}$ sent to the reference generator and the machine model. The schematic of Figure 1 performs current regulation, instantaneous flux weakening operation and compensation of model mismatches at once, as explained with more details in the following Subsections.

\subsection{The machine model}

The machine model contained in the reference generator of Figure 1 is a conventional digital implementation of the voltage equations of a synchronous machine in space-vector notation:

$$
\begin{aligned}
& \boldsymbol{u}_{d q}=R_{s} \boldsymbol{i}_{d q}+\frac{d \lambda_{d q}}{d t}+j \omega_{m e} \lambda_{d q} \\
& \lambda_{d q}=\boldsymbol{f}\left(\boldsymbol{i}_{d q}, \lambda_{p m}\right)
\end{aligned}
$$

where the space vector voltages, currents and flux linkages are denoted as $\boldsymbol{u}_{d q}, \boldsymbol{i}_{d q}$ and $\lambda_{d q}, R_{s}$ is the stator resistance, $\omega_{m e}$ is the mechanical-electrical speed related to the mechanical speed $\omega_{m}$ by the pole pairs number $p$. The vector function $\boldsymbol{f}$ maps the $d q$ flux linkages as function of the $d q$ currents and (if present) the flux contribution of the magnets $\lambda_{p m}$, which is also indicated as a space vector. SPMSMs and IPMSMs exhibit only a $d$-axis contribution of the flux linkage from the magnets. PMaSynRMs show only a $q$-axis contribution. SynRMs, instead, have a zero $\lambda_{p m}$.

In general, $\boldsymbol{f}$ is a non-linear function that accounts for the most important magnetic relations between currents and flux linkages, in particular the magnetic saturation and the crosssaturation effects. For a more detailed discussion on the theoretical foundations of the function $\boldsymbol{f}$, the Reader is redirected to [12].

The implementation of the machine model in Equation (1), with few nomenclature modifications, is shown in Figure 2.

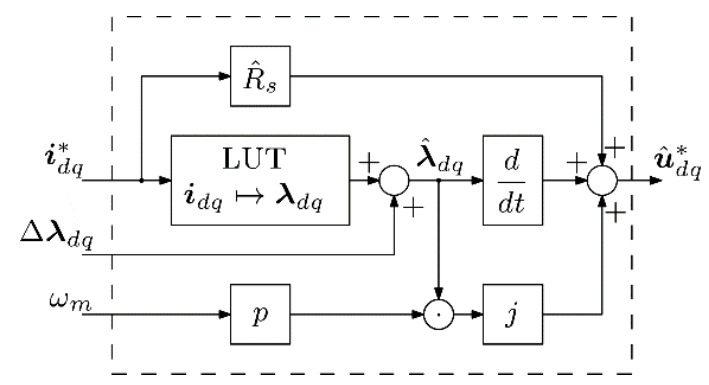

Figure 2: The machine model used in the proposed algorithm.

The modifications are related to the nature of the control implementation in practical cases. The output of the look-up table (LUT) describing the function $\boldsymbol{f}$ generates an estimated flux linkage $\hat{\lambda}_{d q}$. In this work, it is assumed that $\hat{\lambda}_{d q}$ does not necessarily corresponds to the actual flux linkage $\lambda_{d q}$, due to the possible inaccuracies in estimating the function $\boldsymbol{f}$. Although several methods and improvements for an automatic estimation of $\boldsymbol{f}$ have been proposed (see $[11,12]$ and related references), a fully reliable LUT cannot be assumed a priori. For this reason, the value of $\hat{\lambda}_{d q}$ is further modified by the contribution of $\Delta \lambda_{d q}$, which is explained in Subsection 2.3.

Furthermore, the value of the stator resistance is not perfectly known unless an on-line estimator that tracks its changes with the temperature is implemented. Thus, the parameter $R_{S}$ is replaced with the notation $\hat{R}_{S}$. In this work, it is assumed that the stator resistance can be estimated and tracked with injection methods as the one in [13], since this method does not make use of magnetic model parameters which are assumed to be unreliable, as previously discussed. 
A final consideration is required for the derivative operation. Due to the digital nature of the control, the implementation of the derivative operator must be carefully performed to avoid noisy signals. Typical implementations that include a derivative operation followed by low-pass filtering are an established and effective way to circumvent the problem, and will not be discussed further in this paper.

\subsection{The current regulation}

Figure 3 shows a rearrangement of Figure 1 for the current regulation part only, in which the PI regulators block is depicted with its proportional and integral contributions.

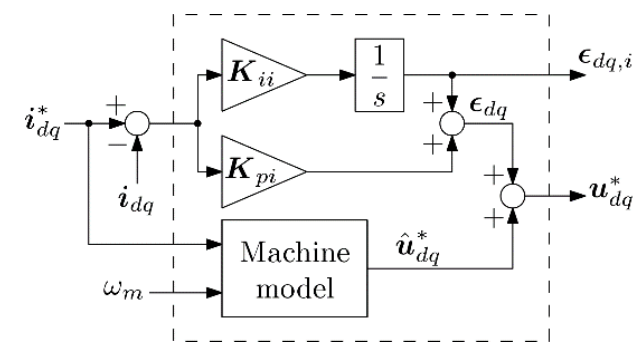

Figure 3: The current regulation with compensating term.

While the contribution $\boldsymbol{\epsilon}_{d q}$ is used to compensate the feedforward action of the estimated reference voltages calculated by the machine model, the signal taken as representative of the model mismatches is the output of the integral part of the PI regulators. This choice follows the same solution previously adopted in [14], where it is shown that any mismatch of magnetic characteristics is visible, in steady-state conditions, in the integral part of the PI regulation. In principle, the whole signal $\boldsymbol{\epsilon}_{d q}$ could be used to represent any model mismatch, but a smoother corrective action is obtained by considering only the integral part. The Reader is redirected to [14] for a more detailed theoretical analysis of this solution.

\subsection{The flux and torque compensation calculation}

Subsection 2.1 showed that the flux linkage estimated by the machine model can be adjusted with a corrective term, which originates somehow from the integral part of the current PI regulators as described in Subsection 2.2. The processing of the signal $\boldsymbol{\epsilon}_{d q, i}$ to obtain $\Delta \boldsymbol{\lambda}_{d q}$ is depicted in Figure 4.

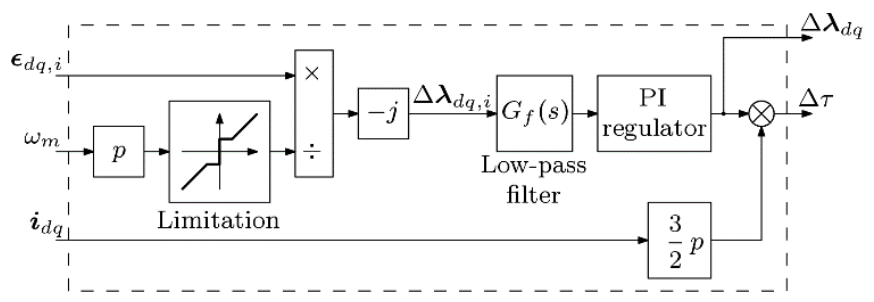

Figure 4: The flux and torque compensation calculator.

Assuming that the stator resistance is well estimated (see discussion in Subsection 2.1) and that the inverter nonlinearities such as pulse-width modulation (PWM) delays and IGBT dead-times are compensated, the machine model implementing Equations (1) at steady-state (without derivative terms) has only one potential inaccuracy, related to the knowledge of the function $\boldsymbol{f}$. As a matter of fact, the contribution of the PI integral part (the proportional part is zero at steady-state) compensates for the mismatch in the flux linkage estimation, such that:

$$
\boldsymbol{\epsilon}_{d q, i}=j \omega_{m e}\left(\boldsymbol{\lambda}_{d q}-\hat{\boldsymbol{\lambda}}_{d q}\right)=j \omega_{m e} \Delta \boldsymbol{\lambda}_{d q}
$$

and, therefore, the flux linkage mismatch is obtainable as:

$$
\Delta \lambda_{d q}=-\frac{j}{\omega_{m e}} \boldsymbol{\epsilon}_{d q, i}
$$

Typically, the division by $\omega_{m e}$ poses some computational issues at lower speeds, but simple countermeasures as a minimum allowed speed (see [14] for further details) can be taken to circumvent the problem. Further low-pass filtering as in Figure 4 ensures a smooth transition. Another important issue is that the compensation as in Equation (3) does not ensure error-free flux linkage estimation at steady-state, and therefore an integral action (or a PI regulation) is required as shown in Figure 4. Further details on this theoretical aspect are included in [14].

Along with the flux linkage compensation, a torque compensation can be calculated by means of the following equation:

$$
\Delta \tau=\frac{3}{2} p \Delta \lambda_{d q} \times i_{d q}
$$

The calculation of the torque compensation is required to avoid a mismatch between the expected torque reference (the value of $\tau^{*}$ produced by a speed regulator) and the actual torque produced by the machine. This problem does not appear when the flux linkage compensation term is equal to zero, but any flux linkage mismatch automatically generates a torque mismatch that affects the tracking of $\tau^{*}$. More details on this aspect will be given in the following Subsection 2.4.

\subsection{The reference generator}

The last, and more complex part, of the proposed control scheme is the reference generator of Figure 1. A more detailed view of its schematic, in form of flow-chart operations, is shown in Figure 5.

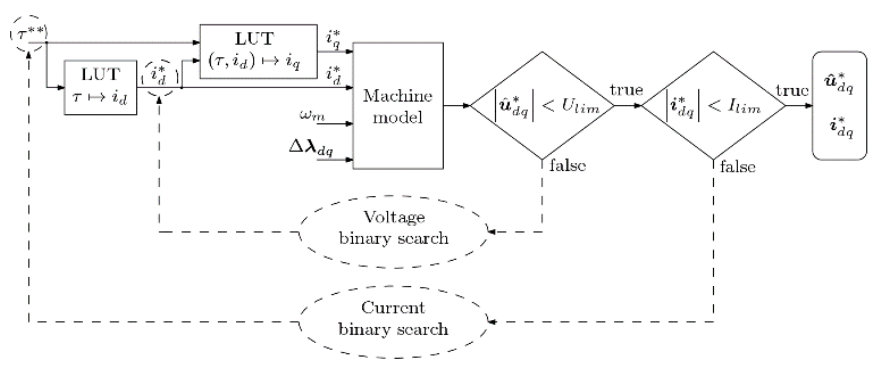

Figure 5: The reference generator in a flow-chart form.

The signal $\tau^{* *}$, obtained by modifying the torque reference $\tau^{*}$ with the torque compensation $\Delta \tau$, is sent to a LUT which provides a corresponding, provisional $d$-axis current reference $i_{d}^{*}$. Among the several possibilities for the generation of $i_{d}^{*}$, a suitable choice is initially made by assuming that the drive will operate according to the MTPA principles, where the magnitude of the current vector is minimised for a given torque. Consequently, the provisional value of $i_{d}^{*}$ is obtained 
following a MTPA trajectory, which is possibly mapped at the commissioning stage where the function $\boldsymbol{f}$ is also mapped (see Subsection 2.1). It is important to underline that any mismatch in the estimation of $\boldsymbol{f}$ leads inevitably to a mismatch of the MTPA trajectory, too.

The provisional $i_{d}^{*}$ is sent through a LUT accepting $\tau^{* *}$ as second input, whose output is a provisional $q$-axis current reference $i_{q}^{*}$. Note that this second LUT does not represent a MTPA trajectory only, but it describes all possible combinations of torque and currents in the $d q$ reference frame. Since the torque is estimated as a cross-product of flux linkages and currents, this second LUT can be considered as a mere inversion of the function $\boldsymbol{f}$, with some related additional processing that can be performed at the commissioning stage. Both provisional current references, as well as $\omega_{m e}$ and $\Delta \boldsymbol{\lambda}_{d q}$, are sent to a machine model of the type shown in Figure 2, to generate two provisional estimated voltages which are immediately tested against the maximum allowed voltage $U_{\text {lim }}$. Two aspects are worth underlining: first, the provisional estimated voltages originate from a machine model which is adjusted by the flux linkage compensation (see Figure 2), therefore they are also corrected. Second, the provisional estimated voltages are not sent to the current regulation yet: they are first evaluated in order to guarantee the imposed voltage limitations.

If the voltage limitation is not guaranteed, i.e. the provisional voltages are greater than $U_{\text {lim }}$, a nested binary search is executed within the same control cycle. A new provisional value of $i_{d}^{*}$ is calculated and provided to the LUT for the generation of a new $i_{q}^{*}$, both of which are used to recalculate the provisional voltage references. Once again, the voltage references are checked against $U_{\text {lim }}$, and the whole process continues until the condition is satisfied. The selection of a new $i_{d}^{*}$ depends on the machine type and its flux weakening capabilities.

Once the voltage limitation is satisfied, however, another binary search for the validation of the current limit $I_{\text {lim }}$ must be performed, as a consequence of the torque mismatch originated from a flux linkage mismatch. When a torque reference signal is first processed to generate $i_{d}^{*}$ and $i_{q}^{*}$, it is expected that the combination of currents and flux linkages represented in the LUTs will generate the requested torque. However, this is not true in presence of a flux linkage mismatch, and therefore the torque compensation $\Delta \tau$ is calculated and added to $\tau^{*}$ to obtain $\tau^{* *}$ and a correct torque tracking. However, $\tau^{* *}$ might potentially point to current values outside the nominal current limitation of the machine, and therefore a nested binary search on the current limitation is required. Should the currents be outside the allowed limits, the torque reference $\tau^{* *}$ will be reduced by the search algorithm to always guarantee the current limit.

The nested binary searches are executed within the same control cycle that generates the modified torque reference $\tau^{* *}$, thus avoiding any external voltage regulation loop (with another possible PI regulator) and providing current and voltage references with instantaneous flux weakening capabilities and parameter mismatch compensation. For the machine used in the experiment and the selected PWM switching frequency (see Subsection 3.1), it was verified that the nested binary searches and the rest of the control were well executed within every control cycle.

\section{Experimental tests}

\subsection{The experimental setup}

A picture of the experimental test bench is reported in Figure 6. The 11-kW SynRM prototype under test, whose parameters are reported in Tab. I, is shown on the right side. The load machine (an 11-kW Baldor machine) is visible on the left side. The load machine is controlled via an off-the-shelf ABB ACS850 converter. The SynRM, instead, is connected only to the power unit of an ABB ACS850, while its control board has been replaced by a custom interface and connected to an OPAL-RT Technologies OP5600 system (the black box on the left upper side of the figure). The OP5600 is equipped with a quad-core Intel DSP processor at $2.4 \mathrm{GHz}$ and a Virtex 6 FPGA, and the selected PWM switching frequency is $4 \mathrm{kHz}$. The phase currents and the DC-bus voltage are measured with a custom measurement box and connected to the $\mathrm{A} / \mathrm{D}$ board of the OP5600. The digital I/O of the OP5600 are used to communicate with the ACS850 power unit through the custom interface.

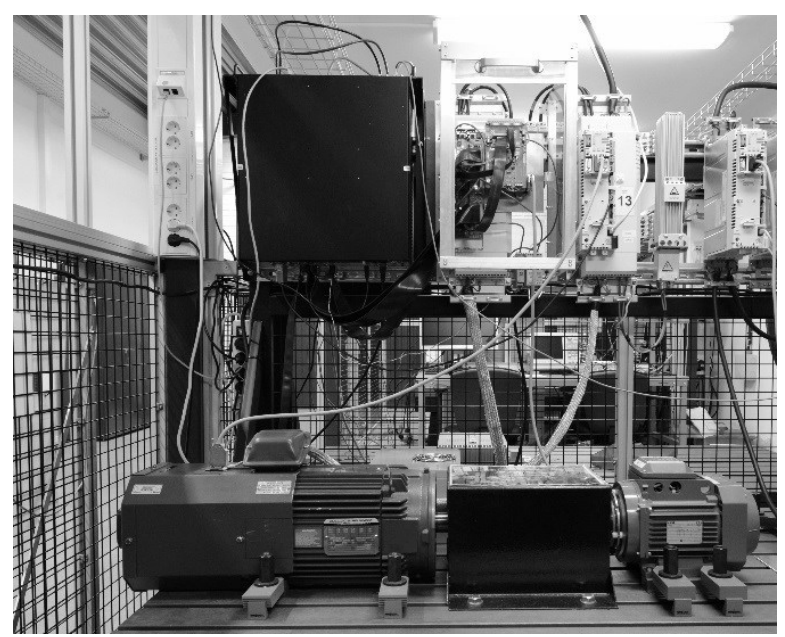

Figure 6: The experimental setup.

\begin{tabular}{|c|c|}
\hline Parameter & Value \\
\hline Nominal power & $11 \mathrm{~kW}$ \\
Nominal speed & $6000 \mathrm{rpm}$ \\
Nominal torque & $17 \mathrm{Nm}$ \\
Pole pairs & 2 \\
Nominal current & $18 \mathrm{~A}$ \\
Nominal voltage & $400 \mathrm{~V}$ \\
Stator resistance & $0.72 \Omega$ \\
Linear d-axis inductance & $80 \mathrm{mH}$ \\
Linear q-axis inductance & $60 \mathrm{mH}$ \\
SynRM mechanical inertia & $0.00351 \mathrm{kgm}$ \\
Total mechanical inertia & $0.034 \mathrm{kgm}$ \\
Viscous friction & $1 \mathrm{e}-2 \mathrm{Nms} / \mathrm{rad}^{2}$ \\
\hline
\end{tabular}

Table 1: SynRM parameters. 

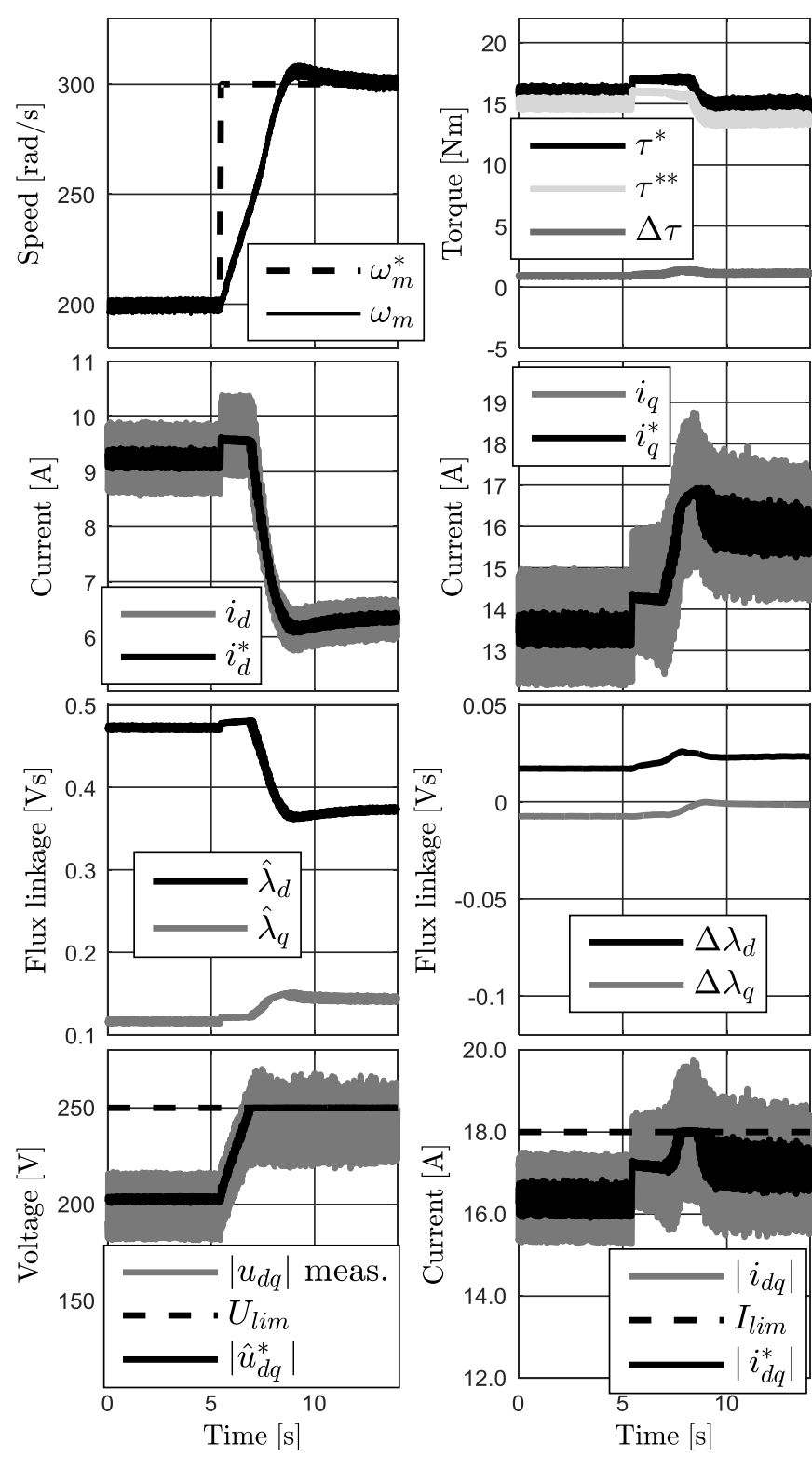

Figure 7: Limitations test with correct magnetic model.

\subsection{The measurements}

Figure 7 and Figure 8 show the test performed with correct and mismatched magnetic model, respectively. A high speed transient is applied in presence of $90 \%$ of load torque, while the voltage limitation was purposely reduced to $250 \mathrm{~V}$ in order to induce a voltage limitation.

Using a correct magnetic model, the contribution of $\Delta \boldsymbol{\lambda}_{d q}$ is negligible (around $5 \%$ of $\lambda_{d q}$ ), and so for $\Delta \tau$, and solely related to imperfections in the model, or in the compensation of PWM delay and dead-times. When the speed transient occurs, the currents and voltages rise as expected. When the voltage limitation is hit, the currents are modified to enter the (early, in this case) flux weakening operation. Since the SynRM has no magnets, the $i_{d}$ and thus $\lambda_{d}$ are correctly reduced to weaken the magnetic field, while $i_{q}$ is increased. It is also noticeable that for a time interval the current limitation has intervened.
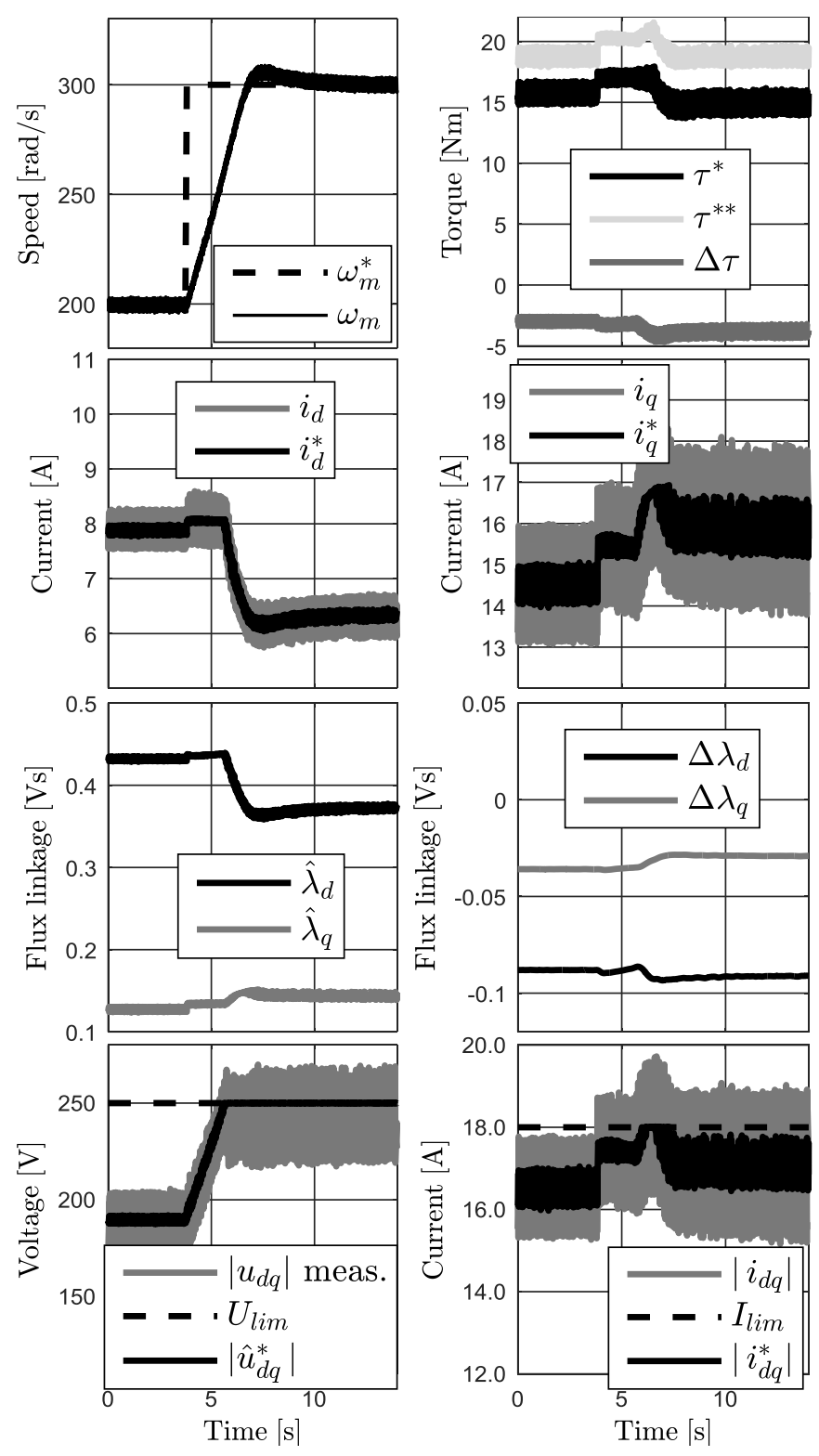

Figure 8: Limitations test with magnetic model mismatch.

In the case of a magnetic model mismatch as in Figure 8, the LUTs used by the machine model and the reference generator were grossly modified, by removing the cross-saturation effect and by overestimating the flux linkage curves by $10 \%$ with respect to the largest curves describing $\lambda_{d}$ and $\lambda_{q}$ as function of the currents. The two curves used as the $\boldsymbol{f}$ function for the test in Figure 8 are shown in Figure 9 as dashed lines, along with the original SynRM curves used for the test in Figure 7 (only three curves of the cross-saturation effect are shown).

Similarly to the results shown in Figure 7, the drive copes correctly with the voltage and the current limitations. However, in this case the contribution of $\Delta \lambda_{d q}$ is in the range of $20 \%$ of the actual $\lambda_{d q}$, actively contributing to the correct estimation of $\hat{\lambda}_{d q}$. The torque compensation is also in the order of $30 \%$ of the actual torque, bringing the drive back to a correct match between the expected load of $15 \mathrm{Nm}$ and the torque reference $\tau^{*}$. Figure 9 shows also the compensated final values of $\lambda_{d}$ and 
$\lambda_{q}$ from Figure 8, marked with crosses, along with the values from the original mismatched LUTs, marked with circles. The control is able to compensate the wrong LUTs estimations.
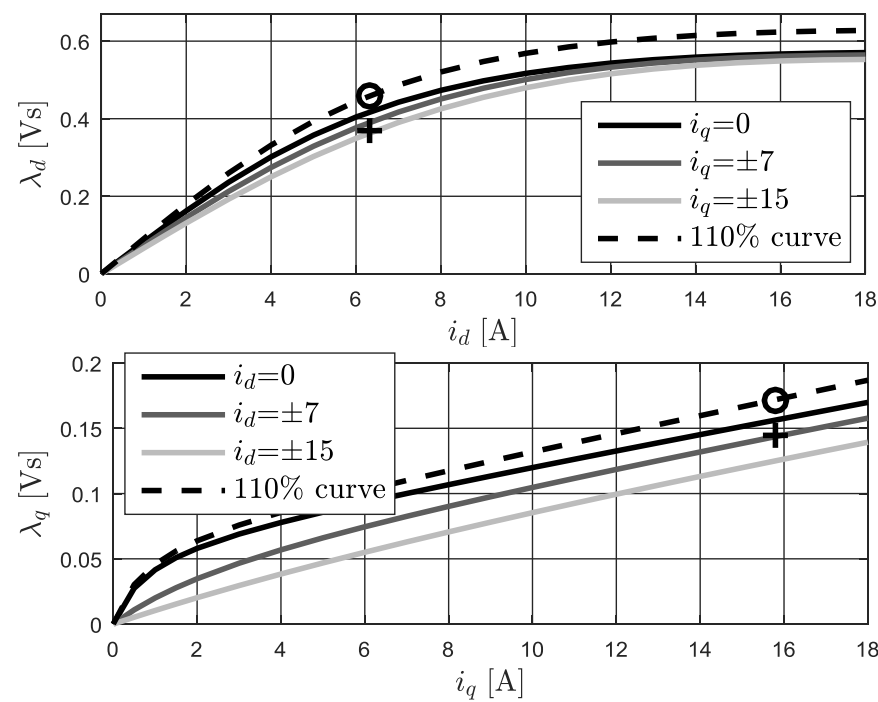

Figure 9: SynRM $\lambda_{d q}$ curves (originals and with mismatch).

\section{Conclusions}

The paper describes an alternative model-based current/torque regulation algorithm for synchronous machines, where an initial magnetic model information is actively used to provide instantaneous flux weakening capabilities and real-time compensation of magnetic parameters mismatch. The goal is achieved by nested binary searches of current and voltage references that fulfil the given constraints, using the compensated magnetic model information. The algorithm has been successfully tested on an 11-kW SynRM prototype, proving the validity of the proposed approach.

\section{References}

[1] J. C. Cintron-Rivera, S. N. Foster, C. A. Nino-Baron, and E. G. Strangas, "High performance controllers for interior permanent magnet synchronous machines using look-up tables and curve-fitting methods," in Proceedings of the IEEE International Electrical Machines and Drives Conference (IEMDC), Chicago, Illinois, USA, May 1215, 2013, pp. 268-275, (2013).

[2] N. Yang, G. Luo, W. Liu, and K. Wang, "Interior permanent magnet synchronous motor control for electric vehicle using look-up table," in Proceedings of the 7th IEEE International Power Electronics and Motion Control Conference (ECCE Asia 2012), Harbin, China, Jun. 2-5, 2012, pp. 1015-1019, (2012).

[3] Y.-D. Yoon, W.-J. Lee, and S.-K. Sul, "New flux weakening control for high saliency interior permanent magnet synchronous machine without any tables," in Proceedings of the European Conference on Power Electronics and Applications, Aalborg, Denmark, Sep. 25, 2007, pp. 1-7, (2007).

[4] S. Huang, Z. Chen, K. Huang, and J. Gao, "Maximum torque per ampere and flux-weakening control for PMSM based on curve fitting," in Proceedings of the IEEE Vehicle Power and Propulsion Conference (VPPC 2010), Lille, France, Sep. 1-3, 2010, pp. 1-5, (2010).

[5] Z. Lei, W. Xuhui, Z. Feng, K. Liang, and Z. Baocang, "Deep field-weakening control of PMSMs for both motion and generation operation," in Proceedings of the 14th IEEE International Conference on Electrical Machines and Systems (ICEMS 2011), Beijing, China, Aug. 20-23, 2011, pp. 1-5, (2011).

[6] G. Kang, J. Lim, K. Nam, H.-B. Ihm, and H.-G. Kim, “A MTPA control scheme for an IPM synchronous motor considering magnet flux variation caused by temperature," in Proceedings of the 19th Annual IEEE Applied Power Electronics Conference and Exposition (APEC), vol. 3, Anaheim, California, USA, Feb. 22-28, 2004, pp. 1617-1621, (2004).

[7] Y.-S. Kim and S.-K. Sul, "Torque control strategy of an IPMSM considering the flux variation of the permanent magnet," in Proceedings of the 42nd IEEE Industry Application Conference Annual Meeting (IAS 2007), New Orleans, Louisiana, USA, Sep. 23-27, 2007, pp. 13011307, (2007).

[8] A. S. Babel, J. C. Cintron-Rivera, and E. G. Strangas, “A multiple look-up table torque controller for improved performance of IPM machines," in Proceedings of the IEEE International Electrical Machines and Drives Conference (IEMDC), Chicago, Illinois, USA, May 1215, 2013, pp. 521-525, (2013).

[9] Y. Deshpande, H. A. Toliyat, W. Xiaoyan, "Standstill position estimation of SPMSM", Proceedings of the 38th Annual Conference on IEEE Industrial Electronics Society (IECON), Montreal, Canada, Oct. 25-28, 2012, pp. 2024-2029, (2012).

[10] E. Armando, R. Bojoi, P. Guglielmi, G. Pellegrino, and M. Pastorelli, "Experimental identification of the magnetic model of synchronous machines," IEEE Trans. Ind. Appl., vol. 49, no. 5, pp. 2116-2125, (2013).

[11] G. Štumberger, B. Polajžer, B. Štumberger, M. Toman, and D. Dolinar, "Evaluation of experimental methods for determining the magnetically nonlinear characteristics of electromagnetic devices," IEEE Trans. Magn., vol. 41, no. 10, pp. 4030-4032, (2005).

[12] L. Peretti, P. Sandulescu, and G. Zanuso, "Quasistandstill self-commissioning of synchronous reluctance machines including cross-magnetisation effects," IET Electric Power Applications, vol. 9, no. 9, pp. 642-651, (2015).

[13] G. Zanuso, L. Peretti, and P. Sandulescu, "Stator reference frame approach for DC injection-based stator resistance estimation in electric drives," in Proceedings of the 11th IEEE International Conference on Power Electronics and Drive Systems (PEDS), Sydney, Australia, Jun. 9-12, 2015, pp. 867-872, (2015).

[14] G. Zanuso, L. Peretti, and M. Zigliotto, "Permanent magnet synchronous machines flux linkage estimation with zero steady-state error and its field-programmable gate array implementation," IET Electric Power Applications, vol. 9, no. 4, pp. 332-343, (2015). 